\title{
The calculation of low voltage solution based on state space search method in ill-conditioned system
}

\author{
A.Shahriari, Student Member, IEEE, H. Mokhlis, Member, IEEE, A. H. A. Bakar, Member, IEEE, \\ M. Karimi, Student Member, IEEE, J. A. Laghari, M. Kazeminezad
}

\begin{abstract}
This paper implements the State Space Search Method (SSSM) in polar coordinate form to calculate low voltage solution and Maximum Loading Point of system in ill conditioned system. SSSM modifies the direction of state variable (buses voltage and phase) by using optimal multiplier in order to converge load flow equations in ill conditioned system. The privilege of SSSM emerges in the keeping dimension of load flow jacobian matrix constant. While another method such as continuation and homotopy methods change the framework of jacobian matrix based on predictor and corrector in term of increasing load demand. Indeed, the calculation process of SSSM is based on standard Newton Raphson load flow method. The validation of SSSM is shown by the testing IEEE test systems of 14 and 30 in well and maximum loading point as ill-conditioned systems.
\end{abstract}

Index Terms-- state space search method, low voltage solution, optimal multiplier, ill conditioned system, maximum loading point.

\section{INTRODUCTION}

$\mathrm{E}$ conomic, Environmental, and technical problems such as the difficulty in construction of new transmission lines, new generation plants, and the raising load demand have let power system to operate near to its limit capacity[1,2]. This is emerged in approaching to Maximum Loading Point (MLP), transmission lines with high $\mathrm{R} / \mathrm{X}$ ratio and bus connections have a very high resistance and very low impedance that causes the power flow ill-conditioned system [3]. Therefore, the chance of the voltage collapse phenomenon is increased due to power system operation in the instability zone [4]. Although, voltage collapse is a dynamic problem, it can be considered as a static issue if the power systems parameters change slowly [5]. The change in these parameters corresponds to a small load increase in power system. Hence, a set of static nonlinear algebraic equations of the power flow equations is considered for the voltage stability study [6]. In this context, a several approaches based on power flow equations have been introduced to determine load flow solutions in ill conditioned system. This case forces the power flow equations to have Low Voltage Solution (LVS) or solution Type-1 as unstable equilibrium operation point.
Under this condition, the original power flow solutions as interesting power flow solution is called High Voltage Solution (HVS) [7].

In this regard, two main approaches are have been presented in order to find power system low voltage solutions, the State Space Search Methods (SSSM) and path following methods (PFM) [7,8]. PFM utilizes trajectory of load demand increasing in PV curves to detect MLP and beyond it [9. This method is powerful and robust for detecting LVS [10]. However, PFM needs a large number of iteration and any prior information of the direction of load increasing to converge [7]. Also, PFM could not handle ill-conditioned and unsolvable condition in appropriate manner [11]. The PFM is classified as the Continuation methods (CM) and the Homotopy methods (HM) $[9,11,12]$. In contrary of CTM, SSSM does not need to several iterations and any previous information of direction of load demand increasing to calculate LVSs [13]. Because, SSSM is based on Newton Raphson Load Flow Method (NRLFM) using optimal multiplier [13,14]. The optimal multiplier as accelerator damper converges the load flow equations in ill conditioned systems [15].

This paper uses the SSSM in polar coordinate from in order to find LVS at MLP of system as ill-conditioned system [16]. The polar coordinate compares with rectangular coordinate is system is more appropriate for power flow solution for illconditioned. Furthermore, polar coordinate form banes switching all of PV type buses to PQ that happened in rectangular form $[17,18,19]$. The contribution of this paper is apparent in introducing the proposed algorithm based on SSSM in order to obtain LVS's and MLP simultaneously. To evaluate the proposed method, case studies based on IEEE test systems of 14 and 30, in well and ill-conditioned systems are tested [20].

\section{IMPROVED STATE SPACE SEARCH METHOD}

The power flow equations as a set of nonlinear equations can be written as:

$$
F(X, H)=0
$$

Where

$X$ - Vector of uncontrolled (state) variables.

$H$ - Vector of controlled variables (active and reactive power of buses). 


\section{$F$ - Load flow function}

The E.q (1) includes only two variables i.e. bus voltage amplitude $\left(X_{a}\right)$ and its angle $\left(X_{b}\right)$ at buses as independent variables in polar coordinate form. Since, Taylor series, expansion of E.q (1) based on these state variables is formulated as follow:

$$
\begin{aligned}
& F\left(X_{a}{ }^{i}+\Delta X_{a}{ }^{i}, X_{b}{ }^{i}+\Delta X_{b}{ }^{i}\right)-F\left(X_{a}{ }^{i}, X_{b}{ }^{i}\right)-\left[\Delta_{X_{a}, X_{b}} F\right]^{i}\left[\Delta X_{a}{ }^{i}, \Delta X_{b}{ }^{i}\right] \\
& -\frac{1}{2}\left[\Delta X_{a}{ }^{i}, \Delta X_{b}{ }^{i}\right]^{T}\left[\Delta^{2}{ }_{X_{a}, X_{b}} F\right]^{j}\left[\Delta X_{a}{ }^{i}, \Delta X_{b}{ }^{i}\right]=0
\end{aligned}
$$

The classical Newton-based methods can solve E.q (2) by neglecting the second term E.q (2). But in ill conditioned system first initial estimation of newton-based methods state variables such as Newton Raphson Load Flow Method (NRLFM) is far away from real solution. In this context, the State Space Search Method (SSSM) modifies the direction of state variables in state space from first initial estimation to find best stable solution or low voltages solutions [7, 13]. Therefore, the modification of next step of state variables is

$$
X^{i+1}=X^{i}+\lambda \Delta X^{i}
$$

where $\lambda$ is multiplier damper that is used to modify the mismatch vector of state variables. Hence, E.q (3) can be rewritten by using $\lambda$

$$
\begin{gathered}
F\left(X_{a}{ }^{i}+\right. \\
\left.\Delta X_{a}{ }^{i}, X_{b}{ }^{i}+\Delta X_{b}{ }^{i}\right)-F\left(X_{a}{ }^{i}, X_{b}{ }^{i}\right)-\lambda^{i}\left[\Delta_{X_{a}, X_{b}} F\right]^{i}\left[\Delta X_{a}{ }^{i}, \Delta X_{b}{ }^{i}\right] \\
-\frac{1}{2} \lambda_{i}{ }^{2}\left[\Delta X_{a}{ }^{i}, \Delta X_{b}{ }^{i}\right]^{T}\left[\Delta^{2}{ }_{X_{a}, X_{b}} F\right]^{i}\left[\Delta X_{a}{ }^{i}, \Delta X_{b}{ }^{i}\right]=0
\end{gathered}
$$

$\lambda$ is called optimal multiplier due to optimization method based on nonlinear programming technique is utilized to calculate it. Under this condition, the multiplier cost function in E.q (5) is defined as objective function in order to obtain then the correction value of state variable as follows

$$
\begin{aligned}
& C=\frac{1}{2} \mid F\left(X_{a}^{i}+\Delta X_{a}{ }^{i}, X_{b}{ }^{i}+\Delta X_{b}{ }^{i}\right)-F\left(X_{a}{ }^{i}, X_{b}{ }^{i}\right)-\lambda^{i}\left[\Delta_{X_{a}, X_{b}} F\right]^{i}\left[\Delta X_{a}^{i}, \Delta X_{b}{ }^{i}\right] \\
& -\frac{1}{2} \lambda_{i}{ }^{2}\left[\Delta X_{a}^{i}, \Delta X_{b}{ }^{i}\right]^{T}\left[\Delta^{2} X_{a}, X_{b} F\right]^{i}\left[\Delta X_{a}{ }^{i}, \Delta X_{b}{ }^{i}\right]=0
\end{aligned}
$$

We can get by manipulating (5) to

$C=\frac{1}{2}\left[D+E \lambda+\lambda^{2} G\right]^{T}\left[D+E \lambda+\lambda^{2} G\right] \rightarrow$

$$
=\frac{1}{2}\left[D^{T} D+2 D^{T} E \lambda+\left(E^{T} E+2 G^{T} D\right) \lambda^{2}+2 E^{T} G \lambda^{3}+G G^{T} \lambda^{4}\right]
$$

where

$$
\begin{aligned}
& D=F\left(X_{a}{ }^{i}+\Delta X_{a}^{i}, X_{b}{ }^{i}+\Delta X_{b}{ }^{i}\right)-F\left(X_{a}{ }^{i}, X_{b}{ }^{i}\right) \\
& B=\left[\Delta_{X_{a}, X_{b}} F\right]^{i}\left\lfloor\Delta X_{a}^{i}, \Delta X_{b}^{i}\right\rfloor \\
& C=\frac{1}{2} \lambda_{i}{ }^{2}\left[\Delta X_{a}^{i}, \Delta X_{b}^{i}\right]^{T}\left[\Delta^{2} X_{a}, X_{b} F\right]^{i}\left[\Delta X_{a}^{i}, \Delta X_{b}^{i}\right]
\end{aligned}
$$

Iba et al. improved that the optimum points of multiplier cost function as quartic function in E.q (6) has a two minimum points and one maximum point [14]. The assumption illustration of E.q (6) is depicted in Fig.1. In well conditioned system, the classical newton-based method can only detect one of these minimum points as interested solution point called the high voltage solution (HVS). For this case $\lambda$ is equal to one. In ill conditioned system, by increasing the load demand of system, two minimum points of E.q (6) are forced to get closer to each other and meet at MLP located at maximum point of Fig.1. For this condition the closet value of $\lambda$ to one is selected as the optimal multiplier to find LVS. Furthermore, it can be observed that E.q (6) is based on NRLFM.

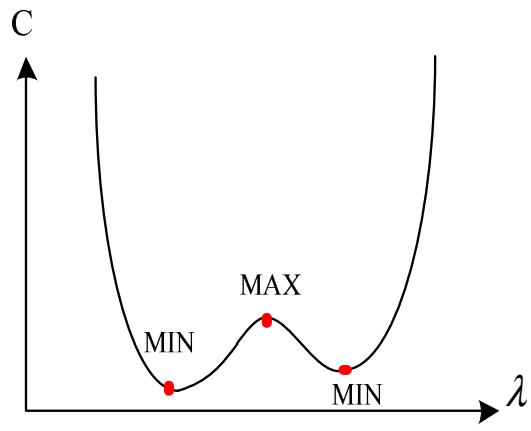

Fig.1. Illustration of assumption multiplier cost function respect $\lambda$

However, optimal multiplier $\lambda$ is computed by minimizing E.q (6) respect with $\lambda$ as follows

$\frac{d L}{d \lambda}=D^{T} E \lambda+\left(D^{T} D+2 G^{T} D\right) \lambda+3 E^{T} G \lambda^{2}+2 G G^{T} \lambda^{3} 0$

where $E=-D$ in order to simplify (7) [13].

The Cardan method is used to find possible real roots of this cubic equation. E.q (7) has three real roots in well-conditioned system. The numbers of real solutions of E.q (7) are decreased at MLP. E.q (7) does not have any real solution in unsolvable operation zone beyond MLP.

The proposed algorithm based on SSSM to calculate LVS in terms of load demand increasing is as follows

Increase predefined load demands based defined step then run NRLFM

1- If NRLFM converges go to 1 otherwise go to 3

2-Calculate the closet optimal multiplier to one of equation (6) by using cardan method due to calculate low voltage solutions.

3- Increase load demands based defined step

4- If that E.q (7) has a real solution (as index to approaching to MLP) stop process and calculate the LVS based on this optimal multiplier otherwise go to (3).

\section{CASE STUDY}

IEEE 14 and 30- bus test system are tested to show convergence characteristics of SSSM at MLP as illconditioned system. The single transmission diagram of IEEE 14 and 30 bus systems are shown in Fig.2 and Fig.3 respectively. The linear active and reactive load demand models are utilized to detect the maximum loading point of these systms as follows [15]:

$$
\begin{aligned}
& P=P_{0}+\alpha P \\
& Q=Q_{0}+\alpha Q
\end{aligned}
$$


where $P_{0}, Q_{0}$ and $P, Q$ are the intial and actual vector of active and reactive powers. Alfa $(\alpha)$ involves the step size and the direction the load demand changing. The calculated MLP based on proposed algorithm with $\alpha=0.001$ for IEEE 14 and 30 bus systems are 3.95135 as well as 2.93124 respectively. The newton-based methods cannot converge in ill conditioned system due to the fact the region of LVS if far away from their initial guess.

The Table I and II show the divergence characteristics of NRLFM in calculating LVS's at MLP IEEE 30 and 14 bus systems respectively. In addition, the drastic difference between load flow solutions in ill and well -conditioned for both systems confirms that the result of NRLFM is not at vicinity of its initial guess in ill conditioned system.

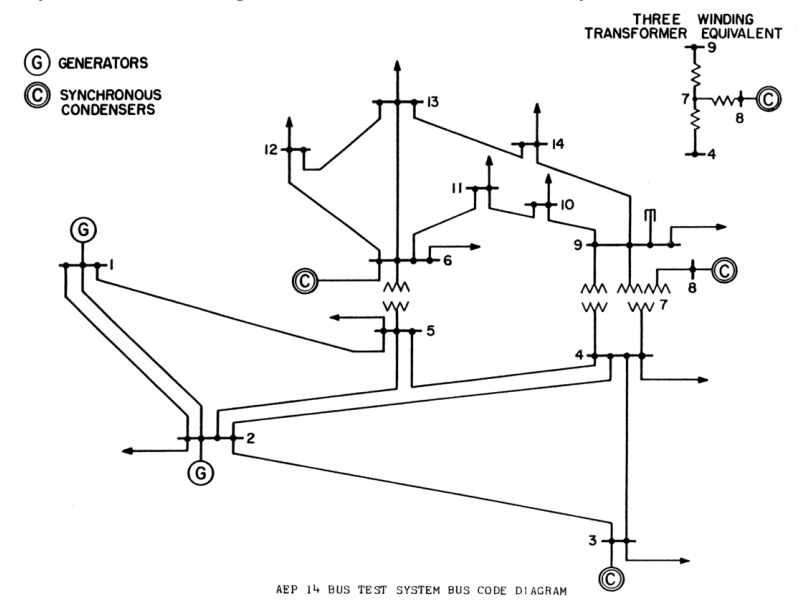

Fig.2. Transmission single line diagram of IEEE 14 bus system

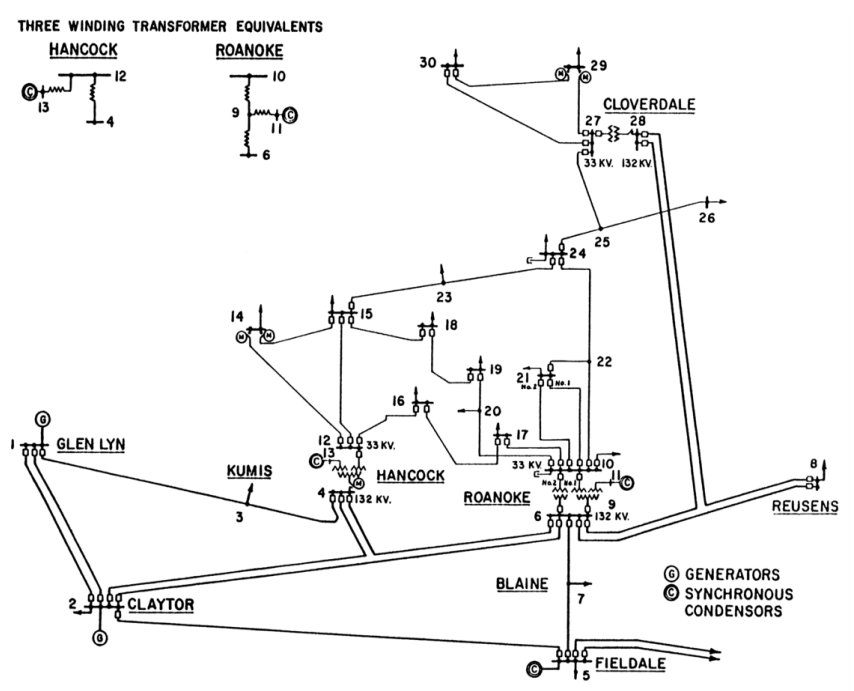

Fig.3. Transmission single line diagram of IEEE 30 bus system

TABLE I

THE PERFORMANCE OF STANDARD NEWTON RAPHSON METHOD FOR IEEE 30 BUS SYSTEM IN WELL AND ILL CONDITIONED SYSTEM

\begin{tabular}{|c|c|c|c|c|}
\hline \multirow{2}{*}{$\begin{array}{c}\text { Bus } \\
\text { Number }\end{array}$} & \multicolumn{2}{|c|}{ Well-conditioned } & \multicolumn{2}{c|}{ Ill conditioned (MLP) } \\
\cline { 2 - 5 } & $\begin{array}{c}\text { Voltage } \\
\text { (P.U) }\end{array}$ & $\begin{array}{c}\text { Angle } \\
\text { (radian) }\end{array}$ & $\begin{array}{c}\text { Voltage } \\
\text { (P.U) }\end{array}$ & $\begin{array}{c}\text { Angle } \\
\text { (radian) }\end{array}$ \\
\hline 1 & 1.06 & 0 & 1.06 & 0 \\
\hline 2 & 1.043 & -0.09343 & 1.043 & -0.47751 \\
\hline
\end{tabular}

\begin{tabular}{|c|c|c|c|c|}
\hline 3 & 1.02098 & -0.13132 & 0.815706 & -0.58657 \\
\hline 4 & 1.01206 & -0.16188 & 0.803409 & -0.75188 \\
\hline 5 & 1.01 & -0.24748 & 1.01 & -1.08467 \\
\hline 6 & 1.01019 & -0.19351 & 0.852351 & -0.90794 \\
\hline 7 & 1.00234 & -0.22487 & 0.885864 & -1.01805 \\
\hline 8 & 1.01 & -0.20661 & 1.01 & -1.00975 \\
\hline 9 & 1.051 & -0.25174 & 0.757811 & -1.27762 \\
\hline 10 & 1.04508 & -0.28089 & 0.576883 & -1.53443 \\
\hline 11 & 1.082 & -0.25174 & 1.082 & -1.27762 \\
\hline 12 & 1.05563 & -0.27122 & 0.753158 & -1.51882 \\
\hline 13 & 1.071 & -0.27122 & 1.071 & -1.51882 \\
\hline 14 & 1.04087 & -0.28636 & 0.640906 & -1.62638 \\
\hline 15 & 1.03656 & -0.28753 & 0.57752 & -1.6264 \\
\hline 16 & 1.04367 & -0.27993 & 0.642334 & -1.5502 \\
\hline 17 & 1.0396 & -0.28431 & 0.571194 & -1.57242 \\
\hline 18 & 1.02741 & -0.29732 & 0.504248 & -1.70584 \\
\hline 19 & 1.02514 & -0.29978 & 0.479262 & -1.72491 \\
\hline 20 & 1.02934 & -0.29605 & 0.497643 & -1.68255 \\
\hline 21 & 1.03259 & -0.28867 & 0.465421 & -1.62291 \\
\hline 22 & 1.0331 & -0.28845 & 0.456753 & -1.62505 \\
\hline 23 & 1.0263 & -0.29342 & 0.404919 & -1.70571 \\
\hline 24 & 1.02101 & -0.29522 & 0.23136 & -1.77448 \\
\hline 25 & 1.01662 & -0.28685 & -0.052947 & -2.0035 \\
\hline 26 & 0.99892 & -0.29419 & -0.7675 & -2.8508 \\
\hline 27 & 1.02243 & -0.27713 & 0.147969 & -1.92636 \\
\hline 28 & 1.00676 & -0.20450 & 0.792666 & -0.93353 \\
\hline 29 & 1.00257 & -0.29864 & -0.150049 & -10.1672 \\
\hline 30 & 0.99108 & -0.31407 & -0.399481 & -23.1452 \\
\hline
\end{tabular}

TABLE II

THE PERFORMANCE OF STANDARD NEWTON RAPHSON METHOD FOR IEEE 14 BUS SYSTEM IN WELL AND ILL CONDITIONED SYSTEM

\begin{tabular}{|c|c|c|c|c|}
\hline \multirow{2}{*}{$\begin{array}{c}\text { Bus } \\
\text { Number }\end{array}$} & \multicolumn{2}{|c|}{ Ill conditioned (MLP) } & \multicolumn{2}{c|}{ Well-conditioned } \\
\cline { 2 - 5 } & $\begin{array}{c}\text { Voltage } \\
\text { (P.U) }\end{array}$ & $\begin{array}{c}\text { Angle } \\
\text { (radian) }\end{array}$ & $\begin{array}{c}\text { Voltage } \\
\text { (P.U) }\end{array}$ & $\begin{array}{c}\text { Angle } \\
\text { (radian) }\end{array}$ \\
\hline 1 & 1.06 & 0 & 1.06 & 0 \\
\hline 2 & 1.045 & 11.322 & 1.045 & -0.087009 \\
\hline 3 & 1.01 & 23.3714 & 1.01 & -0.222258 \\
\hline 4 & 11.0687 & 22.8167 & 1.01777 & -0.180316 \\
\hline 5 & 12.7693 & 18.7376 & 1.02 & -0.153031 \\
\hline 6 & 1.07 & 43.7231 & 1.07 & -0.25882 \\
\hline 7 & 8.19797 & 34.447 & 1.06208 & -0.238405 \\
\hline 8 & 1.09 & 34.447 & 1.09 & -0.238405 \\
\hline 9 & 9.19502 & 39.2377 & 1.05674 & -0.267176 \\
\hline 10 & 8.10829 & 40.8546 & 1.05171 & -0.270678 \\
\hline 11 & 4.70401 & 42.4966 & 1.05733 & -0.26702 \\
\hline 12 & 1.81008 & 43.787 & 1.05521 & -0.273437 \\
\hline 13 & 2.55967 & 2.55967 & 1.05055 & -0.274556 \\
\hline 14 & 7.44001 & 43.6162 & 1.03608 & -0.287826 \\
\hline
\end{tabular}

On the other hand, the value of phase angle at MLP in Table I and II implies the enhancement of buses voltage angle during demand increasing declines the voltage stability margin. Due to voltage stability can be verified as a static issue if the power systems parameters change slowly.

The performance of SSSM to find LVS at MLP is shown in Table III. On account of value of voltage in TABLE III and IV can be assessed that SSSM computes the possible lowest LVS. 
Base on this fact, the role of buses 14 and 30 in IEEE 14 and 30 bus systems as the weakest buses in ill conditioned are same with their roles in well -conditioned of aforesaid test systems. For instance, bus 30 in IEEE 30bus system is selected as the most sensitive bus for contingency analysis for voltage collapse in ill and well- conditioned simultaneously.

TABLE III

THE PERFORMANCE OF STATE SPACE SERACH METHOD FOR IEEE 30 BUS SYSTEM AT MAXIMUM LOADING POINT AS ILL CONDITIONED SYSTEM

\begin{tabular}{|c|c|c|}
\hline Bus number & Voltage (P.U) & $\begin{array}{c}\text { Angle } \\
\text { (radian) }\end{array}$ \\
\hline 1 & 1.06 & 0 \\
\hline 2 & 1.043 & -0.413933 \\
\hline 3 & 0.894945 & -0.513603 \\
\hline 4 & 0.889619 & -0.647211 \\
\hline 5 & 1.01 & -0.961153 \\
\hline 6 & 0.92345 & -0.777013 \\
\hline 7 & 0.929161 & -0.885209 \\
\hline 8 & 1.01 & -0.848162 \\
\hline 9 & 0.915832 & -1.00969 \\
\hline 10 & 0.833729 & -1.14206 \\
\hline 11 & 1.082 & -1.00969 \\
\hline 12 & 0.910295 & -1.11679 \\
\hline 13 & 1.071 & -1.11679 \\
\hline 14 & 0.846481 & -1.18134 \\
\hline 15 & 0.821531 & -1.18365 \\
\hline 16 & 0.850475 & -1.1454 \\
\hline 17 & 0.819988 & -1.16101 \\
\hline 18 & 0.776867 & -1.22829 \\
\hline 19 & 0.762978 & -1.23909 \\
\hline 20 & 0.777088 & -1.21859 \\
\hline 21 & 0.78033 & -1.18114 \\
\hline 22 & 0.780842 & -1.18037 \\
\hline 23 & 0.7615 & -1.21152 \\
\hline 24 & 0.714901 & -1.21968 \\
\hline 25 & 0.689427 & -1.21004 \\
\hline 26 & 0.603362 & -1.26168 \\
\hline 27 & 0.716639 & -1.17582 \\
\hline 28 & 0.905938 & -0.815416 \\
\hline 29 & 0.595928 & -1.32425 \\
\hline 30 & 0.525515 & -1.46208 \\
\hline
\end{tabular}

TABLE IV

THE PERFORMANCE OF STATE SPACE SERACH METHOD FOR IEEE 14 BUS SYSTEM AT MAXIMUM LOADING POINT AS ILL CONDITIONED SYSTEM

\begin{tabular}{|c|c|c|}
\hline Bus number & Voltage (P.U) & Angle (radian) \\
\hline 1 & 1.06 & 0 \\
\hline 2 & 1.045 & -0.801241 \\
\hline 3 & 1.01 & -1.75153 \\
\hline 4 & 0.626277 & -1.48106 \\
\hline
\end{tabular}

\begin{tabular}{|c|c|c|}
\hline 5 & 0.585695 & -1.25234 \\
\hline 6 & 1.07 & -2.3345 \\
\hline 7 & 0.750392 & -2.00689 \\
\hline 8 & 1.09 & -2.00689 \\
\hline 9 & 0.658537 & -2.25809 \\
\hline 10 & 0.687476 & -2.31361 \\
\hline 11 & 0.858552 & -2.33472 \\
\hline 12 & 0.972703 & -2.40185 \\
\hline 13 & 0.919648 & -2.39885 \\
\hline 14 & 0.65116 & -2.47655 \\
\hline
\end{tabular}

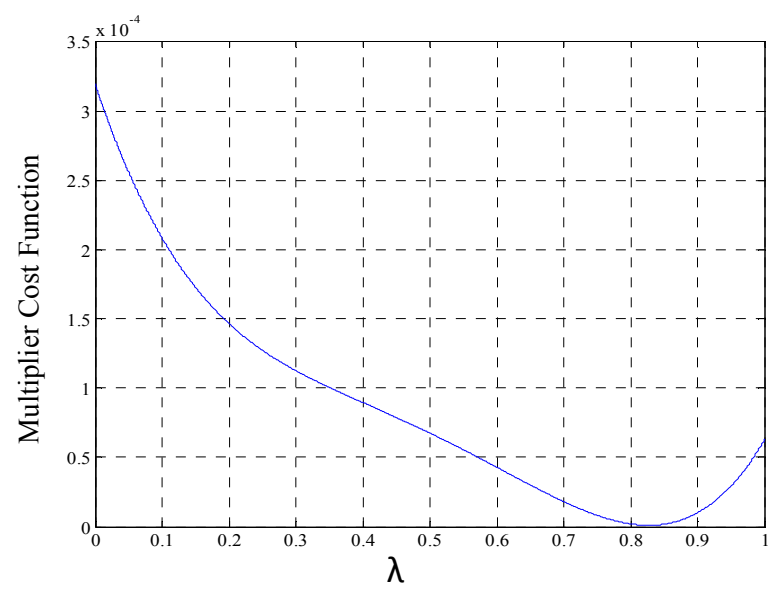

Fig.4 Illustration of multiplier cost function for IEEE 14 bus system

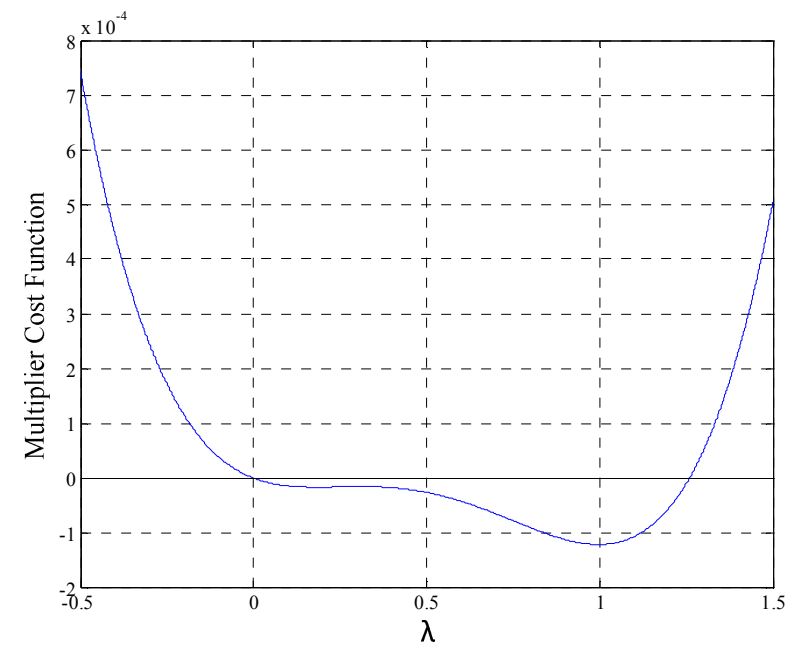

Fig.5 Illustration of multiplier cost function for IEEE 30 bus system

Multiplier cost functions in E.q (6) for 14 and 30 IEEE bus systems are depicted in Fig.4 and Fg.5 respectively. A single minimum point in Fig.4 remarks that only one optimal multiplier remains for IEEE 14 bus system. The value of single optimal multiplier of IEEE 14 bus system is 0.8279903 calculated by E.q (6). Truly, E.q (6) for IEEE 14 bus system has an on real root 0.8279903 and two imaginary roots that are $0.349982+\mathrm{i} 0.212271$ and $0.349982-\mathrm{i} 0.212271$. Furthermore, the existence only on optimal multiplier at MLP approves the LVS's meeting at MLP for IEEE 14 bus system. The close being of 0.8279903 to 1 also is shown. IEEE 30 bus system has the three optimal multiplier at MLP 
consist, $0.117464,0.381862$ and 0.998313 . Indeed, the values of multipliers at minimum points of IEEE 30 bus system multiplier cost function are the optimal multipliers. For this case, the closest optimal multiplier to one is chosen as the desirable optimal multiplier. It can be seen the existence of multi-optimal multiplies at MLP. The important point in this context is that the value of their corresponding multiplier cost functions is very near to each other. This is because the scale of 14 and 30 IEEE bus systems multiplier cost functions are based on 0.0001 .

\section{CONCLUSION}

This paper shows the state space search method (SSSM) is a robust, reliable and simple method in order to calculate Low Voltage solution (LVS) at Maximum Loading Point (MLP) in ill conditioned system. Also, it has been revealed that the proposed algorithms based on SSSM can obtain LVS'S and MLP simultaneously. For this purpose, the closet optimal multiplier to one has been selected as the desirable optimal multiplier to find LVS's at MLP. The capability of SSSM has been validated by IEEE test systems of 14 and 30 in well and maximum loading point as ill-conditioned system.

\section{REFERENCES}

[1] B. Stott, "Review of load-flow calculation methods", Proc. IEEE, vol. 62 n. 1, , pp. 916-929.July 1974.

[2] Wang. Y, da Silva. L.C.P and Wilsun Xu, Investigation of the relationship between ill-conditioned power flow and voltage collapse, IEEE Power Engineering Review, pp. $43-45$ vol.20 n.4, July 2000.

[3] Felix F. Wu "Theoretical Study Of The Convergence of The Fast Decoupled Load Flow", IEEE Trans. Power Syst, vol. 96 n.2, January 1977.

[4] Y. Tamura, H. Mori and S. Iwamoto, "Relationship Between Voltage Instability and Multiple Load Flow Solutions in Electric Power Systems", IEEE Trans. Power App .Syst., vol. 102 n. 5 pp. 1115 - 1125, May 1983.

[5] A. Z. de Souza, C. A. Cañizares, and V. H. Quintana, "New Techniques to Speed Up Voltage Collapse Computations Using Tangent Vector", IEEE Trans. Power Syst, vol. 12 n.3, pp, 1380-1387August 1997.

[6] Ning Xie, Ettore Bompard, Roberto, Napoli Franco Torelli "Widely convergent method for finding solutions of simultaneous nonlinear equations". Electric Power Systems Research journal Volume 83, Issue 1, Pages 1-266 February 2012.

[7] R. B. L. Guedes, L. F. C. Alberto and N. G. Bretas, Power system lowvoltage solutions using an auxiliary gradient system for voltage collapse purposes, IEEE Trans. Power Syst, vol. 20 n.3 pp1528-1537, August 2005.

[8] F. Milano, Continuous Newton's Method for Power Flow analysis, IEEE Trans. Power Syst, Vol. 24 n.1, pp. 50-57, February 2009.

[9] Shahriari, A. ; Bakar, A.H.A. ; Mokhlis, H. "Comparative studies on Non-Divergent Load flow methods in well, ill and unsolvable condition" IEEE Conference Power System Technology (POWERCON), 2010.

[10] Shao-Hua Li and Hsiao-Dong Chiang, "Continuation Power Flow With Nonlinear Power Injection Variations: A Piecewise Linear Approximation", IEEE Trans. Power Syst, vol. 23 n.4 pp. 1637 - 1643., November 2008.

[11] Y. Chen and C. Shen, “ A Jacobian-free Mewton-GMRES(m) method with adaptive preconditioner and its application for power flow calculations" IEEE Trans. Power Syst., vol. 21 n. 3, August .2006, pp. 1096-1103.

[12] Yorino, N. Hua-Qiang Li Sasaki, H, A predictor/corrector scheme for obtaining Q-limit points for power flow studies, IEEE Trans. Power Syst, vol.20 n. 2, F, pp.130-137, February 2005.

[13] S. Iwamoto and Y. Tamura, A load flow calculation method for ill conditioned power systems, IEEE Trans. Power App. Syst., vol. 100 n .3, , pp. 1736-1743,April1981.
[14] K. Iba, H. Suzuki, M. Egawa, and T.Watanabe, A method for finding a pair of multiple load flow solutions in bulk power systems, IEEE Trans. Power Syst., vol. 5 n.2, pp. 582-591, May 1990.

[15] T. J. Overbye and R. P. Klump, Effective calculation of power system low -voltage solutions, IEEE Trans. Power Syst., vol. 11 n.1 pp.75-82 ., February 1996.

[16] M. D. Schaffer and D. J. Tylavsky, A nondiverging polar form Newtonbased power flow, IEEE Trans. Ind. App., vol. 24 n.l, pp.870877.September/October 1988.

[17] L. M. C. Braz, C. A. Castro, and C. A. F. Murari, A critical evaluation of step size optimization based load flow methods, IEEE Trans. Power Syst., vol. 15 n.1, , pp. 202-207, February 2000

[18] J. E. Tate and T. J. Overbye, A comparison of the optimal multiplier in polar and rectangular coordinates, IEEE Trans. Power Syst., vol. 20 n 4. , pp. 1667-1674, November 2005.

[19] Huang,W.-T.;Yao,K.-C, "New network sensitivity-based approach for real time complex power flow calculation" IET Generation, Transmission \& Distribution Vol. 6, iss. 2, pp. 109-12. 2012.

[20] Available: www.ee.washington.edu/research/pstca. 